\title{
Efficacy and safety of combined doxofylline and salbutamol in treatment of acute exacerbation of chronic obstructive pulmonary disease
}

\author{
Xianrong Du (1), Hongju Bao² (1), Daguo Zhao ${ }^{3 *}$ (1)
}

\begin{abstract}
SUMMARY
OBJECTIVE: The aim of this study was to investigate the efficacy and safety of combined doxofylline and salbutamol in the treatment of acute exacerbation of chronic obstructive pulmonary disease.

METHODS: A total of 68 acute exacerbation of chronic obstructive pulmonary disease patients were randomly divided into control group (34 cases) and experimental group (34 cases), who received the doxofylline treatment and combined doxofylline and salbutamol treatment for 1 week, respectively. During the treatment, the remission time of typical respiratory manifestations was recorded, and the adverse reactions were observed. At the end of treatment, the treatment efficacy was evaluated. Before and after treatment, the pulmonary function indexes and serological indicators were detected.

RESULTS: After treatment, compared with control group, in experimental group, the effective rate of treatment was significantly increased $(p<0.05)$, the remission time of typical respiratory manifestations was significantly shortened $(p<0.05)$, the pulmonary function indexes were significantly improved $(p<0.05)$, the serum high-sensitivity C-reactive protein and cystatin C levels were significantly decreased, respectively $(p<0.05)$, and the serum prealbumin level was significantly increased $(p<0.05)$. In addition, the adverse reaction rate had no significant difference between two groups ( $p>0.05$ ).

CONCLUSIONS: In the treatment of acute exacerbation of chronic obstructive pulmonary disease, the combined use of doxofylline and salbutamol can quickly relieve the respiratory symptoms, mitigate the pulmonary dysfunction, and reduce the inflammatory response, thus promoting the outcome of patients.

KEYWORDS: Doxofylline. Salbutamol. Chronic obstructive pulmonary disease. Inflammation.
\end{abstract}

\section{INTRODUCTION}

Chronic obstructive pulmonary disease (COPD) is a kind of airway inflammatory disease with high morbidity and mortality. It is one of the common respiratory diseases in the elderly. Acute exacerbation of COPD (AECOPD) is an important event in the clinical process of COPD. It can be caused by nonstandard treatment in stable period (e.g., discontinuation of relevant inhaled drug treatment), changes in environmental and physical and chemical factors (e.g., air pollution and smoking), respiratory tract infection, and so on ${ }^{1}$. The typical manifestations of AECOPD are the increased cough, increased sputum volume and/or purulent or mucinous purulent sputum, and aggravated dyspnea ${ }^{2}$. The onset process of AECOPD will not only accelerate the deterioration of lung function, but also increase the risk of death in the future. At present, most commonly used drugs for AECOPD include bronchodilators (e.g., $\beta 2$ receptor agonists

\footnotetext{
'Shanxi Provincial People's Hospital, Emergency Department - Taiyuan, China.

${ }^{2}$ Sozhou Ninth People's Hospital, Department of Respiratory Medicine - Suzhou, China.

${ }^{3}$ The First Affiliated Hospital of Soochow University, Department of Critical Care Medicine - Suzhou, China.

*Corresponding author: zhaodgsz@126.com

Conflicts of interest: the authors declare there are no conflicts of interest. Funding: none.

Received on June 07, 2021. Accepted on July 20, 2021.
} 
and theophylline), anti-inflammatory drugs (e.g., glucocorticoids), and other drugs (e.g., expectorants), among which the bronchodilators are basic drugs ${ }^{3}$. Doxofylline is a kind of theophylline drug that can relieve the asthma, relax the bronchial smooth muscle, and improve the respiratory muscle function. It is a commonly used bronchodilator in the treatment of AECOPD ${ }^{4}$. Salbutamol is a short-acting $\beta 2$ receptor agonist. It can relax the airway smooth muscle, reduce the wheezing, relieve the bronchospasm, and improve the gas exchange. It is another common bronchodilator for rapid control of AECOPD symptoms ${ }^{5}$. In this study, the combined doxofylline and salbutamol was applied for the treatment of AECOPD, and the efficacy and safety of this strategy was observed. This study will provide a basis for further application of this treatment strategy.

\section{METHODS}

\section{General clinical data}

A total of 68 patients with AECOPD treated in our hospital from July 2018 to November 2019 were selected as the research objects. They were randomly divided into control group and experimental group, 34 cases in each group. In the control group, there were 19 males and 15 females. The age was $52-80$ years, with mean age of $66.68 \pm 8.38$ years. The course of disease was $6-14$ years, with mean course of $9.80 \pm 2.41$ years. As for pulmonary function grade in Global Initiative for Chronic Obstructive Lung Disease (GOLD), there were 5 cases of grade I, 8 cases of grade II, 14 cases of grade III, and seven cases of grade IV. In the experimental group, there were 16 males and 18 females. The age was 54-79 years, with mean age of $64.06 \pm 6.29$ years. The course of disease was $5-15$ years, with mean course of $7.26 \pm 3.82$ years. There were 7 cases of GOLD grade I, 7 cases of grade II, 11 cases of grade III, and nine cases of grade IV. There was no significant difference of gender, age, disease course, or GOLD grade between two groups ( $p>0.05$ ). This study was approved by the ethics committee of Shanxi Provincial People's Hospital. Written informed consent was obtained from all participants.

\section{Inclusion criteria and exclusion criteria}

The inclusion criteria were as follows:

(a) the patients met the diagnostic criteria of AECOPD;

(b) the age was less than 80 years; and

(c) the patients presented the aggravated dyspnea, fever, cough, expectoration, and other symptoms.

The exclusion criteria were as follows:

(a) the patients had allergic history to doxofylline or salbutamol; (b) the patients were complicated with diseases not suitable for use of salbutamol;

(c) the patients were complicated with mental disorders;

(d) the patients were complicated with rheumatic immune diseases;

(e) the patients were complicated with tumor, liver or kidney dysfunction, or heart failure;

(f) the patients had respiratory surgery history;

(g) the patients were diagnosed with pneumothorax, pulmonary embolism, pneumonia, or other diseases; and

(h) the patients had contraindications of pulmonary function examination.

\section{Treatment strategies}

The patients in two groups were asked to take light diet and to avoid tobacco, wine, and sour or spicy food. They received the routine resolving phlegm and anti-infection treatment. In control group, the patients were injected with doxofylline injection $(0.2 \mathrm{~g}$ doxofylline was dissolved in $100 \mathrm{ml}$ of $0.9 \%$ sodium chloride solution) by intravenous drip, twice a day, for one week. In experimental group, based on the intravenous drip of doxofylline injection in the control group, the patients received the atomization inhalation of salbutamol (2.5 $\mathrm{mg}$ salbutamol sulfate was diluted into $4 \mathrm{ml}$ of $0.9 \%$ sodium chloride solution), twice (morning and evening) a day, for one week.

\section{Evaluation of total treatment efficacy}

During the treatment, the remission time of typical respiratory manifestations, such as wheezing, cough, and expectoration, in two groups was recorded, and the adverse reactions were observed. At the end of treatment, the treatment efficacy was evaluated as follows:

(a) ineffective: the symptoms of wheezing, cough, expectoration, and others were not relieved, or even aggravated further;

(b) effective: the symptoms of wheezing, cough, expectoration, and others were relieved to a certain extent; and

(c) remarkably effect: the symptoms of wheezing, cough, expectoration, and others disappeared.

The effective rate was calculated as follows: effective rate $(\%)=([$ number of effective cases+number of markedly effective cases $] /$ total case number) $\times 100 \%$.

\section{Pulmonary function examination}

Before and after treatment, the patients received the routine pulmonary function examination. The forced expiratory volume in $1 \mathrm{~s}$ (FEV1) and forced vital capacity (FVC) were measured. The ratio of FEVl/FVC was calculated. 


\section{Detection of serological indicators}

Before and after treatment, $5 \mathrm{ml}$ of fasting venous blood was drawn from the patients. The serum high-sensitivity C-reactive protein (hs-CRP) level was measured by immunoturbidimetric method. The serum cystatin $\mathrm{C}(\mathrm{Cys} C)$ and prealbumin levels were measured by automatic biochemical analyzer.

\section{Statistical analysis}

SPSS 20.0 statistical software was used. Measurement data were expressed as mean \pm standard deviation and analyzed using t-test. Enumeration data were expressed as number or rate, and analyzed using $\chi^{2}$ test. $\mathrm{p}<0.05$ presented statistically significant difference for comparison.

\section{RESULTS}

\section{Total treatment efficacy}

At the end of treatment, the number of remarkably effective, effective, and ineffective cases in control group were 16,10 , and 8 , respectively, and that in experimental group were 20,13 , and 2 , respectively. The effective rate in experimental group was $94.12 \%$, which was significantly higher than $76.47 \%$ in control group $\left(\chi^{2}=4.221 ; \mathrm{p}<0.05\right)$.

\section{Remission time of typical respiratory manifestations}

The remission time of typical respiratory manifestations including wheezing, cough, and expectoration in the treatment process was observed. As shown in Table 1, each index in experimental group was obviously shorter than that in control group $(\mathrm{p}<0.05)$.

\section{Pulmonary function indexes}

As shown in Table 2, there was no significant difference in FEV1 or FEV1/FVC between control and experimental groups before treatment $(p>0.05)$. After treatment, FEV1 and FEV1/FVC in each group were significantly higher than those before treatment, respectively $(\mathrm{p}<0.05)$, and each index in experimental group was significantly higher than that in control group $(\mathrm{p}<0.05)$.

\section{Serological indicators}

Before treatment, the serum hs-CRP, CysC, and prealbumin levels had no significant difference between control and experimental groups, respectively ( $p>0.05)$. After treatment, in each group the serum hs-CRP and CysC levels were significantly lower than those before treatment, respectively $(\mathrm{p}<0.05)$, and the serum prealbumin level was significantly higher than that before treatment $(\mathrm{p}<0.05)$. Compared with control group, in experimental group the serum hs-CRP and $\mathrm{Cys} C$ levels were significantly decreased, respectively $(p<0.05)$, and the serum prealbumin level was significantly increased $(\mathrm{p}<0.05$; Table 3$)$.

\section{Adverse reactions}

During the treatment, one case of dizziness, one case of nausea, and one case of dry mouth were found in the control group, and the adverse reaction rate was $8.82 \%$. In the experimental group, two cases of dizziness, one case of nausea, and two cases of dry mouth were found, and the adverse reaction rate was $11.76 \%$. The adverse reaction rate had no significant difference between two groups $\left(\chi^{2}=0.159\right.$, $\left.\mathrm{p}>0.05\right)$.

Table 1. Comparison of remission time of typical respiratory manifestations between two groups.

\begin{tabular}{l|c|c|c|c} 
Group & $\mathrm{n}$ & Wheezing (days) & Cough (days) & Expectoration (days) \\
\hline Control & 34 & $5.05 \pm 1.04$ & $5.77 \pm 1.23$ & $6.45 \pm 1.38$ \\
\hline Experimental & 34 & $4.29 \pm 1.45$ & $4.95 \pm 1.05$ & $5.53 \pm 1.13$ \\
\hline $\mathrm{t}$ & & 2.483 & 2.957 & 3.008 \\
\hline $\mathrm{p}$ & & 0.016 & 0.004 & 0.004 \\
\hline
\end{tabular}

Table 2. Comparison of pulmonary function indexes between two groups.

\begin{tabular}{l|c|c|c|c}
\multirow{2}{*}{ Group } & \multicolumn{2}{|c|}{ FEV1 $(\%)$} & \multicolumn{2}{c}{ FEV1/FVC } \\
\cline { 2 - 5 } Control & Before treatment & After treatment & Before treatment & After treatment \\
\hline Experimental & $43.62 \pm 11.65$ & $52.10 \pm 12.44^{\mathrm{a}}$ & $55.05 \pm 6.23$ & $60.15 \pm 7.83^{\mathrm{a}}$ \\
\hline $\mathrm{t}$ & $44.16 \pm 8.10$ & $68.27 \pm 14.32^{\mathrm{a}}$ & $56.41 \pm 8.10$ & $68.28 \pm 5.41^{\mathrm{a}}$ \\
\hline $\mathrm{p}$ & 0.222 & 4.971 & 0.776 & 4.981 \\
\hline
\end{tabular}

FEVl: forced expiratory volume in $1 \mathrm{~s}$; FVC: forced vital capacity. ${ }^{\mathrm{P}} \mathrm{p}<0.05$ compared with before treatment. 
Table 3. Comparison of serological indicators between two groups.

\begin{tabular}{l|c|c|c|c|c|c}
\multirow{2}{*}{ Group } & \multicolumn{2}{|c|}{$\mathrm{hs-CRP}(\mathrm{mg} / \mathrm{L})$} & \multicolumn{2}{c|}{ CysC $(\mathrm{mg} / \mathrm{L})$} & \multicolumn{2}{c}{ Prealbumin $(\mathrm{mg} / \mathrm{L})$} \\
\cline { 2 - 7 } & $\begin{array}{c}\text { Before } \\
\text { treatment }\end{array}$ & $\begin{array}{c}\text { After } \\
\text { treatment }\end{array}$ & $\begin{array}{c}\text { Before } \\
\text { treatment }\end{array}$ & $\begin{array}{c}\text { After } \\
\text { treatment }\end{array}$ & $\begin{array}{c}\text { Before } \\
\text { treatment }\end{array}$ & $\begin{array}{c}\text { After } \\
\text { treatment }\end{array}$ \\
\hline Control & $42.56 \pm 6.04$ & $28.32 \pm 4.82^{\mathrm{a}}$ & $1.43 \pm 0.28$ & $1.30 \pm 0.18^{\mathrm{a}}$ & $126.21 \pm 23.62$ & $144.93 \pm 28.12^{\mathrm{a}}$ \\
\hline Experimental & $44.18 \pm 7.26$ & $15.62 \pm 3.90^{\mathrm{a}}$ & $1.51 \pm 0.22$ & $1.18 \pm 0.26^{\mathrm{a}}$ & $119.04 \pm 19.80$ & $172.40 \pm 31.22^{\mathrm{a}}$ \\
\hline $\mathrm{t}$ & 1.000 & 11.944 & 1.310 & 2.213 & 1.356 & 3.812 \\
\hline $\mathrm{p}$ & 0.321 & 0.000 & 0.195 & 0.030 & 0.180 & 0.000 \\
\hline
\end{tabular}

hs-CRP: high-sensitivity C-reactive protein; CysC: cystatin C. ${ }^{\mathrm{p}} \mathrm{p}<0.05$ compared with before treatment.

\section{DISCUSSION}

Bronchodilators are the cornerstone of drug therapy for AECOPD $^{6}$. The combination of bronchodilators with different action mechanism and action time is an important treatment strategy for AECOPD. In this study, the combined doxofylline and salbutamol was applied to treatment of AECOPD, for improving the treatment efficacy and reduce the risk of adverse reactions. Results showed that, after 1 week of treatment, compared with single use of doxofylline, in the group using combined doxofylline and salbutamol, the effective rate of therapy was significantly increased, the remission time of typical respiratory manifestations was significantly shortened, and the pulmonary function was significantly improved. In addition, the adverse reaction rate had no significant difference between two treatment strategies. This suggests that, the combined use of doxofylline and salbutamol can play a synergistic and complementary role in treating AECOPD through their different action mechanism, while not reducing the safety of medication.

COPD is mainly caused by chronic nonspecific inflammatory response in trachea, bronchial mucosa, and surrounding tissues. AECOPD is a serious deterioration of COPD at its acute onset ${ }^{7}$. The severe pulmonary infection is an important factor for AECOPD. Therefore, monitoring the inflammatory biomarkers is very helpful for early diagnosis and efficacy evaluation of $\mathrm{AECOPD}^{8}$. hs-CRP is a common inflammatory factor. Its level usually increases significantly when the infection and trauma occur, which belongs to the instinctive response of the body. hs-CRP can be used as an important indicator to predict inflammation and infection'. CysC is a protein with a relative molecular weight of $13.3 \times 10^{3}$, which can effectively reflect the degree of inflammation ${ }^{10}$. Studies have shown that, the serum hs-CRP and CysC levels significantly increase in AECOPD, and they significantly decrease after the treatment. Moreover, the serum hs-CRP and CysC levels in the acute stage of COPD are significantly higher than those in the stable stage of COPD, indicating that hs-CRP and $\mathrm{Cys} C$ are correlated with $\mathrm{AECOPD}^{11,12}$. Prealbumin is a kind of plasma transporter, which can remove the toxic metabolites and effectively repair the tissue cells. When the body has systemic inflammatory response, the prealbumin level will decrease rapidly ${ }^{13}$. It is found that the prealbumin can also be used as inflammatory indicator for early diagnosis of AECOPD. The change trend and degree of prealbumin level can be used to judge the treatment efficacy and prognosis of patients ${ }^{14}$. In our study, after treatment, in each group, the serum hs-CRP and CysC levels were significantly lower than those before treatment, and the serum prealbumin level was significantly higher than that before treatment. This suggests that, both single use of doxofylline and combined use of doxofylline and salbutamol can reduce the inflammatory response in AECOPD patients. Compared with control group, in experimental group, the serum hs-CRP and $\mathrm{Cys} C$ levels were significantly decreased, and the serum prealbumin level was significantly increased. This indicates that, the combined use of doxofylline and salbutamol can further reduce the inflammatory response, compared with single use of doxofylline, which may be related with its better treatment efficacy.

\section{CONCLUSIONS}

To sum up, the application of combined doxofylline and salbutamol in the treatment of AECOPD has definite treatment efficacy. This strategy can quickly relieve the respiratory symptoms, mitigate the pulmonary dysfunction, and reduce the inflammatory response, thus promoting the outcome of patients. This study still has some limitations. First, other action mechanisms related to the therapeutic effect of combined doxofylline and salbutamol have not yet been investigated. Second, the number of patients involved in this study is relatively small. These issues should be solved in subsequent studies to obtain more credible conclusions.

\section{AUTHORS" CONTRIBUTIONS}

DZ: Conceptualization, Writing - review \& editing. XD: Data curation, Writing - original draft. HB: Formal Analysis, Writing - review \& editing. 


\section{REFERENCES}

1. Singh JM, Palda VA, Stanbrook MB, Chapman KR. Corticosteroid therapy for patients with acute exacerbations of chronic obstructive pulmonary disease: a systematic review. Arch Intern Med. 2002;162(22):2527-36. https://doi.org/10.1001/ archinte.162.22.2527

2. Matkovic Z, Huerta A, Soler N, Domingo R, Gabarrús A, Torres $A$, et al. Predictors of adverse outcome in patients hospitalised for exacerbation of chronic obstructive pulmonary disease. Respiration. 2012;84(1):17-26. https:// doi.org/10.1159/000335467

3. Chuang C. Transition of patients with COPD across different care settings: challenges and opportunities for hospitalists. Hosp Pract (1995). 2012;40(1):176-85. https://doi.org/10.3810/ hp.2012.02.958

4. Cazzola M, Calzetta L, Rogliani P, Page C, Matera MG. Impact of doxofylline in COPD: A pairwise meta-analysis. Pulm Pharmacol Ther. 2018;51:1-9. https://doi.org/10.1016/j. pupt.2018.04.010

5. Balint B, Watz $H$, Amos C, Owen R, Higgins M, Kramer B, et al. Onset of action of indacaterol in patients with COPD: comparison with salbutamol and salmeterol-fluticasone. Int J Chron Obstruct Pulmon Dis. 2010;5:311-8. https://doi. org/10.2147/copd.s12120

6. Ko FW, Chan KP, Hui DS, Goddard JR, Shaw JG, Reid DW, et al. Acute exacerbation of COPD. Respirology. 2016;21(7):115265. https://doi.org/10.1111/resp. 12780

7. Rohde G, Borg I, Wiethege A, Kauth M, Jerzinowski S, Dinh $T A D$, et al. Inflammatory response in acute viral exacerbations of COPD. Infection. 2008;36(5):427-33. https://doi.org/10.1007/ s15010-008-7327-5

8. Chen H, Song Z, Qian M, Bai C, Wang X. Selection of diseasespecific biomarkers by integrating inflammatory mediators with clinical informatics in AECOPD patients: a preliminary study. J Cell Mol Med. 2012;16(6):1286-97. https://doi. org/10.1111/j.1582-4934.2011.01416.x

9. Kallergis EM, Manios EG, Kanoupakis EM, Mavrakis HE, Kolyvaki SG, Lyrarakis GM, et al. The role of the postcardioversion time course of hs-CRP levels in clarifying the relationship between inflammation and persistence of atrial fibrillation. Heart. 2008;94(2):200-4. https://doi.org/10.1136/ hrt.2006.108688

10. Okura T, Jotoku M, Irita J, Enomoto D, Nagao T, Desilva $V R$, et al. Association between cystatin $C$ and inflammation in patients with essential hypertension. Clin Exp Nephrol. 2010;14(6):584-8. https://doi.org/10.1007/s10157-010-0334-8

11. Jing Z, Chun C, Ning S, Hong Z, Bei H, Wan-Zhen Y. Systemic Inflammatory Marker CRP Was Better Predictor of Readmission for AECOPD Than Sputum Inflammatory Markers. Arch Bronconeumol. 2016;52(3):138-44. English, Spanish. https:// doi.org/10.1016/j.arbres.2015.01.011

12. Zhang M, Fu SH, Cui H, Zhu BP, Liu L, Wang DL. Serum cystatin $\mathrm{C}$ and indices of lung function in elderly Chinese men with chronic obstructive pulmonary disease. Aging Clin Exp Res. 2014;26(2):193-9. https://doi.org/10.1007/s40520-013-0150-6

13. Dickson PW, Howlett GJ, Schreiber G. Metabolism of prealbumin in rats and changes induced by acute inflammation. Eur J Biochem. 1982;129(2):289-93. https:// doi.org/10.1111/j.1432-1033.1982.tb07051.x

14. Mohan A, Arora S, Uniyal A, Poulose R, Luthra K, Pandey RM, et al. Evaluation of plasma leptin, tumor necrosis factor- $\alpha$, and prealbumin as prognostic biomarkers during clinical recovery from acute exacerbations of chronic obstructive pulmonary disease. Lung India. 2017;34(1):3-8. https://doi. org/10.4103/0970-2113.197101 